\title{
Diseño e Implementación de una Plataforma Automatizada de Gestión de Transacciones P2P con Dinero Electrónico
}

\section{(Design and Implementation of an Automated Management Platform for P2P Transactions with Electronic Money)}

\author{
Andrés Alcocer ${ }^{1}$, Gonzalo Olmedo ${ }^{1}$
}

\begin{abstract}
Resumen:
El dinero electrónico es un medio de pagos implementado por el Banco Central del Ecuador, el cual permite a las personas realizar transacciones comerciales desde su celular; este se puede utilizar para efectuar pagos en grandes establecimientos comerciales denominados "macro agentes", entre los que se encuentran las cadenas de supermercados, pero también se puede utilizar para realizar consumos en todo tipo de negocios particulares como restaurantes, tiendas, etc., mediante transferencias de "persona a persona" denominadas P2P. Sin embargo, el sistema de dinero electrónico, SDE, tal como está concebido induce ciertas desventajas competitivas entre los negocios particulares y los macro agentes; la principal radica en que los primeros no cuentan con un sistema que les permita llevar registro de los cobros efectuados con dinero electrónico. El presente artículo detalla la creación de una plataforma tecnológica que permite actualmente a todo tipo de negocios y personas llevar registro de sus transacciones P2P, mediante una solución automatizada con capacidad de generar balances de los pagos recibidos, los cuales se pueden visualizar fácilmente a través de Internet. Para esto se llevó a cabo un análisis profundo del funcionamiento del SDE, con el fin de establecer una solución eficiente al problema, procurando siempre optimizar recursos.
\end{abstract}

Palabras clave: Dinero electrónico, soluciones inteligentes, persona a persona, P2P, gestión de pagos electrónicos.

\begin{abstract}
:
Electronic money is a payment tool implemented by the Ecuador's Central Bank, it allows people to make commercial transactions using their cellphones; it can be used to pay into big chain stores like supermarkets, known as "macro agents", but it also can be employed for the consumption of goods and services into particular businesses such as restaurants or small shops by using "person to person" transfers known as P2P. However, the particular businesses present some disadvantages compared to the macro agents, the main one is the lack of a system that allows them to have a record of the transactions made with electronic money. This article details the design and implementation of a technological platform that allows all types of businesses and people to keep records of their P2P transactions into an automated system with the ability of generating payment's balance sheets which can be easily viewed through Internet. A research was conducted in order to look for the most efficient technical solution to optimize the available resources. The use of this platform as an external tool extends the capabilities currently provided by the electronic money system.
\end{abstract}

Keywords: Electronic money, smart solutions, person to person, P2P, electronic payments management.

\footnotetext{
${ }^{1}$ Universidad de las Fuerzas Armadas - ESPE, Sangolquí - Ecuador (\{eaalcocer, gfolmedo\} @espe.edu.ec)
} 


\section{Introducción}

\subsection{Antecedentes}

Durante siglos se han establecido un sinfín de medios de comercio, desde el arcaico trueque, hasta formas de dinero intangible que circulan a través de redes computarizadas y pueden dar la vuelta al mundo en tan solo segundos (Beltrán, 2014), todo gracias al desarrollo de la tecnología, que ha supuesto el más grande salto en la forma en que concebimos las transacciones comerciales hoy en día (Guadamuz, 2003).

En nuestro país el Banco Central del Ecuador, BCE, en el año 2014 implementó el Sistema de Dinero Electrónico, SDE (Chao \& Ruiz, 2014) como un medio de pago, el cual está vigente y es gestionado privativamente de conformidad con lo establecido en el Código Orgánico Monetario y Financiero (Asamblea Nacional del Ecuador, 2014); dicho sistema tiene una gran perspectiva de desarrollo, ya que se proyecta moverá al menos 10 millones de dólares anualmente, durante sus primeros años de funcionamiento (Ministerio Coordinador de Política Económica, 2013). Es así, que a julio de 2015 se registraron en Ecuador 47.456 cuentas activas de dinero electrónico, y el monto circulante en el sistema ascendía a 645.669 dólares (M2M Money Payments Latam, 2015).

Por otro lado, en el Ecuador existen más de 15.3 millones de líneas de telefonía celular activas (ARCOTEL, 2015), equivalentes a casi el 100\% de la población nacional, sin embargo en una realidad contrastante, aproximadamente el $40 \%$ de la población económicamente activa, PEA, no está incluida financieramente (Banco Central del Ecuador, 2015); es decir, no forma parte del sistema financiero nacional. Tomando en cuenta que la exclusión del sistema financiero formal ha sido una de las barreras que ha impedido a gran parte de nuestra sociedad superar la pobreza, es un reto urgente encontrar modelos innovadores para extender los servicios financieros hacia estos sectores. El sistema de dinero electrónico del BCE, tiene como objetivo incluir a una población estimada de 2'800.000 ciudadanos (Junta de Regulación Monetaria y Financiera, 2014).

\subsection{Estado del Arte}

El dinero electrónico es un medio de pagos que permite a las personas realizar transacciones comerciales desde cualquier dispositivo móvil celular, sin la necesidad de contar con Internet, saldo, SMS (Short Message Service), o con una cuenta en una entidad financiera. El sistema de dinero electrónico es administrado por el BCE conforme a la estructura mostrada en la Figura 1 (Banco Central del Ecuador, 2015), a continuación. 


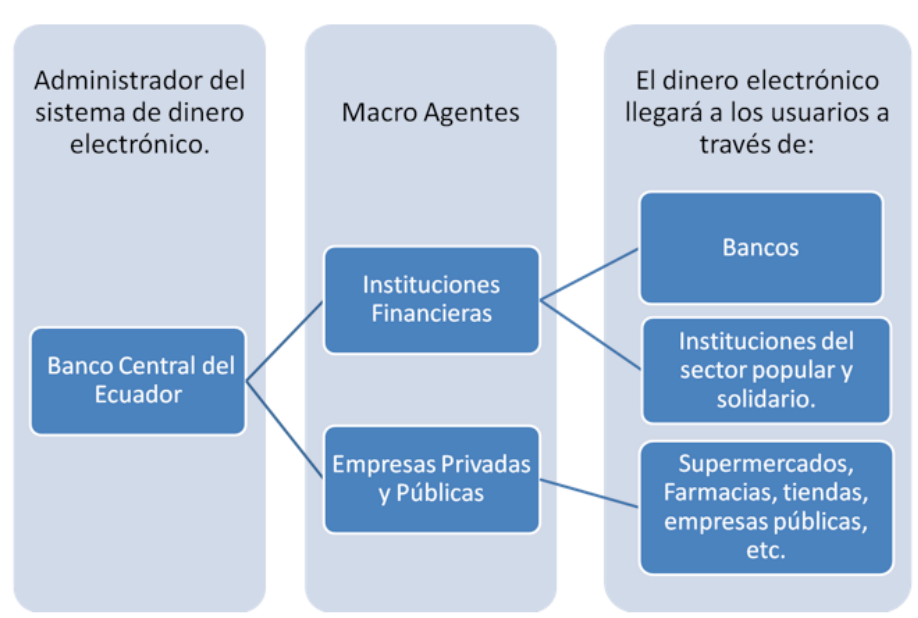

Figura 1. Estructura del Sistema de Dinero Electrónico.

El sistema de dinero electrónico del BCE, funciona sobre una infraestructura tecnológica centralizada, invisible para el usuario; las transacciones se realizan mediante el servicio USSD (Unstructured Supplementary Service Data) de la red celular, y la confirmación de estas se efectúa vía SMS. Para empezar a utilizar el sistema es necesario abrir voluntariamente una cuenta de dinero electrónico denominada "monedero electrónico"; cabe resaltar que este está vinculado con el número telefónico de la línea y no con el dispositivo celular propiamente dicho. Desde la apertura de la cuenta, la totalidad de las operaciones del sistema se realizan a través su interfaz de usuario, a la cual se accede marcando el número *153\# desde cualquier móvil activo.

En el monedero electrónico una persona puede manejar un monto máximo de 9000 dólares (Junta de Regulación Monetaria y Financiera, 2014). Como medida de seguridad el sistema solicita un PIN de 4 dígitos para completar cualquier transacción. Las operaciones que se pueden realizar utilizando el monedero son ilustradas en la Figura 2, donde se muestra como un usuario del SDE puede realizar pagos y transferencias en los establecimientos de los macro agentes (supermercados, farmacias, etc.), pero también puede realizar y recibir pagos a/de otros usuarios del SDE, mediante transferencias de persona a persona, P2P, las cuales permiten que el dinero electrónico pueda ser utilizado como un medio de pago en todo tipo de negocios particulares cuyo dueño posea un monedero activo, como en el caso de restaurantes, almacenes, tiendas, etc.

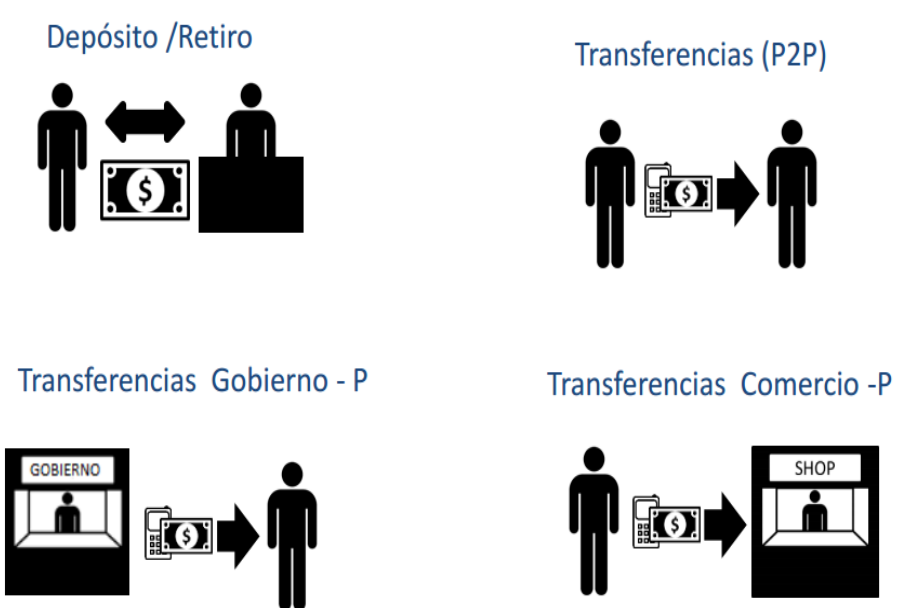

Figura 2. Tipos de Transacciones con Dinero Electrónico. 
Los sistemas de pago de "persona a persona", P2P, han acaparado una parte importante de las transacciones comerciales en la coyuntura actual, es así que ligados a la evolución tecnológica, estos han presentado un gran desarrollo en los últimos años, desde las ya tan comunes transferencias bancarias, hasta los sistemas de pago móviles de última generación, estas soluciones pretenden establecerse en el mercado de consumo basando su estrategia en la omnipresencia tecnológica y costos de servicio más bajos que los sistemas monetarios convencionales. La utilidad que presentan para las personas junto con la comodidad de su uso, han permitido que su aceptación sea masiva y ha impulsado a que germinen paulatinamente distintos tipos de sistemas entre los que destacan los siguientes:

- Sistemas Centralizados Bancarizados: Este es el sistema de pagos P2P más común en el Ecuador, y es brindado por las instituciones financieras formales (bancos, cooperativas, mutualistas, etc) que permiten a sus usuarios realizar transferencias directas e interbancarias. Si bien este tipo de sistemas facilita la circulación de los capitales local e internacionalmente, no tiene la capacidad de integrarse a un entorno de pagos en línea, tampoco todos los bancos admiten transacciones móviles, y finalmente este tipo de transferencias, en especial las interbancarias, pueden tomar varios días en completarse, dificultando el intercambio de bienes o servicios entre sus usuarios.

- Sistemas Centralizados no Bancarizados: Se trata de sistemas administrados de forma centralizada que permiten realizar pagos en sitios web, así como transferir dinero entre usuarios registrados, el ejemplo más destacado de este tipo de sistemas es la plataforma PayPal. Este tipo de plataformas tienen la ventaja de poder integrarse con facilidad a casi cualquier tienda o negocio en línea que desee recibir pagos directos; su principal desventaja es que al no ser un sistema local se requiere de una tarjeta de crédito internacional para transferir los montos al sistema, dichos pagos se direccionan a la plataforma centralizada con sede en el exterior, incurriendo en el correspondiente cobro del impuesto a la salida de capitales.

- Sistemas Criptográficos: Se trata de tipos de cambio alternativos a la moneda circulante oficial de cualquier país, su valor fluctúa de acuerdo a la oferta y la demanda y no tiene respaldo físico en alguna institución bancaria; su medio de acceso es estrictamente digital y se realiza a través de la red mediante claves criptográficas. Al no estar regulado por ente de control o autoridad alguna, este tipo de sistema no genera confianza entre los usuarios por lo que es generalmente relegado para transacciones en el mercado negro. El ejemplo más conocido de este sistema es BitCoin.

- Sistemas Híbridos Colaborativos: Es el caso del sistema de transacciones P2P implementado por el Banco Central del Ecuador, en el cual el dinero está respaldado por dicha institución y regulado por el ente de control pertinente. Al tratarse de un sistema centralizado local, interconectado por las operadoras móviles celulares, el circulante es transferido en tiempo real de usuario a usuario sin la necesidad de que estos posean una cuenta en alguna institución financiera; además los costos son muy bajos en comparación con las transferencias bancarias convencionales. Sin embargo la integración del SDE para pagos en línea esta relegada a menos de 5 instituciones públicas y casi ninguna empresa privada, este es uno de los motivos para desarrollar la presente plataforma de gestión. 


\subsection{Problemática}

La utilización del sistema de dinero electrónico como medio de pagos incentiva sin duda la inserción al sistema financiero de sectores productivos históricamente excluidos, ya que proporciona una alternativa de fácil acceso, generando de esta manera un impacto positivo en el desarrollo económico de la sociedad. Sin embargo, el sistema de dinero electrónico tal como está concebido induce ciertas desventajas competitivas entre los negocios particulares y los macro agentes, la principal radica en que los primeros no cuentan con un sistema que les permita llevar registro de los cobros efectuados con dinero electrónico, tal como se ilustra en las Figuras 3 y 4 , las cuales hacen referencia a una transacción realizada en los dos tipos de establecimientos.

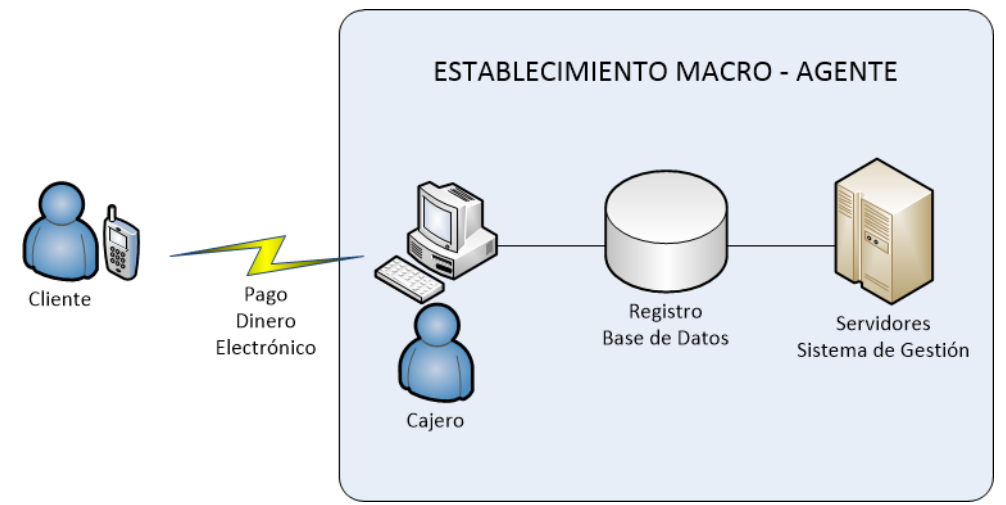

Figura 3. Pago con Dinero Electrónico en un Establecimiento Macro Agente.

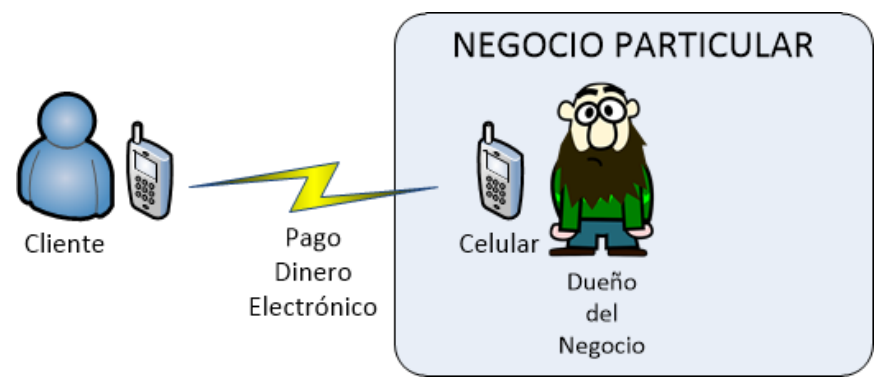

Figura 4. Pago con Dinero Electrónico en un Negocio Particular - P2P.

La Tabla 1 a continuación, muestra un resumen de las diferencias entre los pagos realizados a un macro agente y los pagos realizados vía transacciones $\mathrm{P} 2 \mathrm{P}$ a un negocio particular.

Tabla 1. Diferencias entre Tipos de Pago

\begin{tabular}{|l|l|}
\hline \multicolumn{1}{|c|}{ Establecimiento Macro Agente } & \multicolumn{1}{c|}{ Negocio Particular (P2P) } \\
\hline $\begin{array}{l}\text { Cuenta con un sistema informático interconectado con } \\
\text { el sistema de dinero electrónico del BCE. }\end{array}$ & $\begin{array}{l}\text { No posee un sistema de gestión de cobros con } \\
\text { dinero electrónico. }\end{array}$ \\
\hline $\begin{array}{l}\text { Los pagos se registran de manera automática en una } \\
\text { base de datos. }\end{array}$ & $\begin{array}{l}\text { El único registro de los pagos es un SMS de } \\
\text { confirmación enviado al celular del dueño del } \\
\text { negocio. }\end{array}$ \\
\hline $\begin{array}{l}\text { El sistema informático permite llevar balances de las } \\
\text { transacciones realizadas. }\end{array}$ & $\begin{array}{l}\text { Si se requiere llevar un balance se debe realizar } \\
\text { de forma manual con la información de los SMS } \\
\text { de confirmación. }\end{array}$ \\
\hline $\begin{array}{l}\text { Los reportes de las transacciones son confiables y y } \\
\text { seguros ya que son generados automáticamente por un } \\
\text { sistema informático. }\end{array}$ & $\begin{array}{l}\text { Los reportes manuales son menos confiables y } \\
\text { nada seguros, ya que intervienen personas para } \\
\text { registrarlos y generarlos. }\end{array}$ \\
\hline
\end{tabular}




\subsection{Alcance}

Destacando la importancia de los negocios particulares como actores dinámicos de la economía ecuatoriana, a partir de la problemática analizada, y con el objetivo de plantear una solución a las desventajas del actual sistema de dinero electrónico respecto de los pagos P2P, el presente artículo detalla la creación de una plataforma tecnológica que permita a todo tipo de negocios y personas llevar un registro de sus transacciones P2P, mediante una solución automatizada con la capacidad de generar balances de los pagos recibidos, los cuales se puedan visualizar fácilmente a través de Internet. Para esto se realizó previamente un profundo análisis de la estructura y funcionamiento del sistema de dinero electrónico del Banco Central del Ecuador, y se desarrolló una investigación comparativa entre distintas soluciones tecnológicas con el fin de establecer la alternativa más eficiente, procurando siempre optimizar recursos de una manera sistemática pero a la vez creativa, enfatizando en la seguridad de la información y la escalabilidad del sistema.

\section{Metodología}

La metodología elegida para la creación de la plataforma tecnológica fue el prototyping (prototipado), el cual se concibe como un modelo sistemático para la creación de aplicaciones, utilizado generalmente en el ámbito de la ingeniería de software, pero aplicable a un sin número de áreas. El prototipado es particularmente útil en casos como el presente estudio, en el que se conoce el enfoque general que deberá tener el sistema, pero no se pueden identificar a ciencia cierta los requisitos detallados de entrada, procesamiento o salida. El prototipado consiste en la aplicación secuencial de 4 procesos específicos descritos a continuación:

Investigación Inicial: Es la recopilación documentada de información, en este caso de la estructura y funcionamiento del sistema de dinero electrónico del BCE detallada en la Sección 1, a partir de la cual se pudo identificar la problemática existente, cuyo planteamiento permitió establecer el enfoque general hacia una posible solución, que consiste en la creación de una plataforma tecnológica que haga posible el registro automatizado de transacciones $\mathrm{P} 2 \mathrm{P}$.

Definición de Requerimientos: Con una perspectiva más amplia acerca del enfoque del proyecto, se definirán de manera específica las funcionalidades y características que deberá tener la plataforma, así como los requerimientos técnicos que conformarán su infraestructura. Es importante destacar que a pesar de que en la investigación inicial se identificó un problema evidentemente objetivo, los procesos subsiguientes como la definición de requerimientos, y el diseño del sistema, tienen un carácter subjetivo, cuya orientación dependerá ineludiblemente del autor, quien apegado a la metodología planteará la solución más conveniente según su criterio.

Diseño del Sistema: Con pleno conocimiento de las características funcionales requeridas para la plataforma, se procederá a establecer la arquitectura física y lógica en base a la cual se diseñarán cada uno de los componentes. Con el fin de plantear una solución eficiente, robusta, y 
de bajo costo, se considerarán aspectos como: los distintos tipos de tecnologías existentes, la seguridad de la información, los costos de implementación y mantenimiento, la compatibilidad entre herramientas de software, la escalabilidad del sistema, y la accesibilidad del usuario.

Implementación: A partir de las tecnologías seleccionadas para el diseño de cada componente se realizará de forma sistemática y documentada el proceso de implementación del sistema; para lo cual cada elemento de software y hardware deberá ser meticulosamente calibrado con el fin de cumplir su función específica en coordinación con el resto de elementos.

\subsection{Definición de Requerimientos}

El punto de partida para realizar el registro de los pagos P2P son los SMS de confirmación recibidos en el dispositivo móvil al completar una transferencia monetaria, estos mensajes contienen información relevante de la transacción realizada, la cual incluye el código de referencia único de cada pago, el monto de dinero transferido, y el saldo actual del monedero electrónico; implícitamente se incluyen también la fecha y hora de arribo del SMS, así como el número del remitente, que será siempre el "153" correspondiente al BCE. Un ejemplo de la estructura del mensaje de confirmación se muestra en la Figura 5.

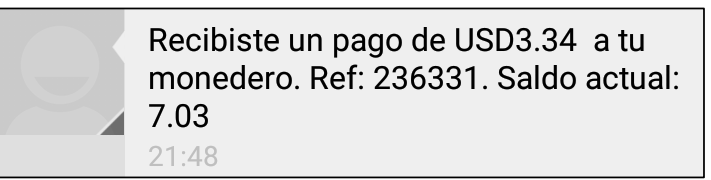

Figura 5. SMS de Confirmación de Pago P2P.

La plataforma deberá estar equipada al menos con un dispositivo móvil, encargado de recibir los mensajes de confirmación; este dispositivo será configurado para actuar como un SMS Gateway, de tal forma que pueda enviar la información de los pagos hacia un servidor remoto que procesará los SMS, y separará los datos relevantes almacenándolos en una Base de Datos. La información de los pagos registrados será utilizada para generar balances, que serán presentados al usuario a través de una interfaz web vía Internet, para lo cual se deberá montar un servidor con su correspondiente arquitectura física y lógica, garantizando además su acceso a la red.

Se ha concebido que existan en el sistema dos tipos de interfaces, una para "usuarios normales" y otra de tipo "administrador"; se contempla estos dos tipos de cuentas como requerimientos de la plataforma considerando que un negocio puede tener varios empleados (usuarios normales), que sean responsables de recibir pagos en determinadas circunstancias, pero generalmente poseen un solo dueño o administrador. Un usuario normal podrá registrar a su nombre cualquiera de los pagos recibidos en la plataforma, lo hará introduciendo el código de referencia correspondiente a dicha transacción, de manera que en sus balances aparecerán solamente los pagos registrados a su nombre, mientras que un administrador tendrá la capacidad de visualizar reportes detallados de 
toda la recaudación (pagos registrados y no registrados). Se podrá o no hacer uso de ambos tipos de cuentas dependiendo de los requerimientos específicos de cada negocio.

El sistema deberá ser escalable, por lo tanto tendrá la capacidad de administrar más de un dispositivo móvil de recaudación si es necesario, por ejemplo, en el caso de un negocio que cuente con varias sucursales y cada una maneje un monedero electrónico donde se desee recibir pagos; de esta forma a pesar que los datos se generen en distintos dispositivos, la plataforma asociará la información a un mismo negocio. La Figura 6 resume las etapas que comprenden el desarrollo de la plataforma, partiendo de los requerimientos planteados.

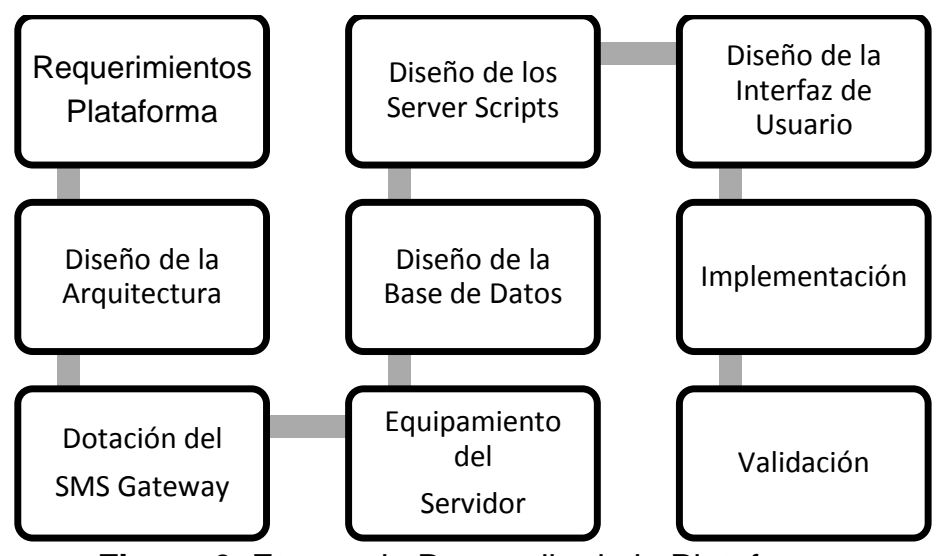

Figura 6. Etapas de Desarrollo de la Plataforma.

\subsection{Diseño del Sistema}

Tomando en consideración los requerimientos planteados en la sección anterior, como primer aspecto de diseño del sistema se establecerán tanto su arquitectura física como su infraestructura lógica, las cuales han sido concebidas con el objetivo de satisfacer las necesidades de funcionamiento proyectadas para la plataforma, así permitirán implementar posteriormente sus distintas funcionalidades. El diseño correspondiente se ilustra en las Figuras 7 y 8 , a continuación.

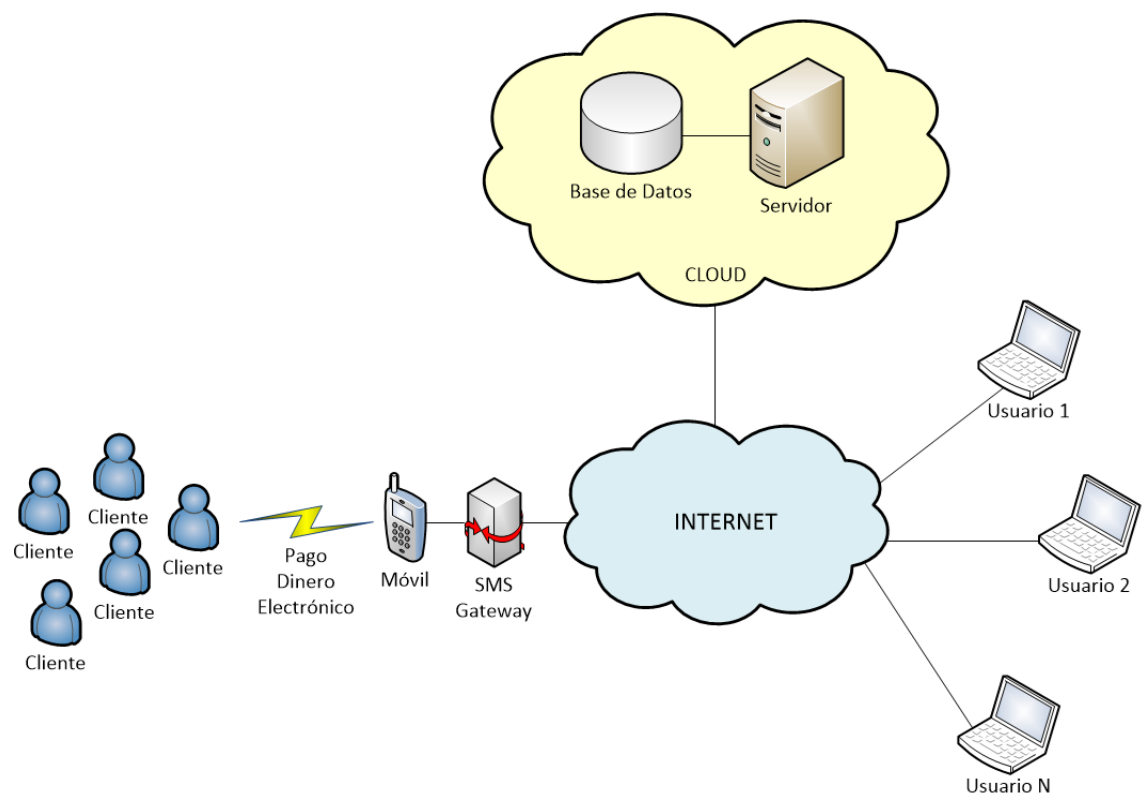

Figura 7. Arquitectura Física - Diseño de Hardware 


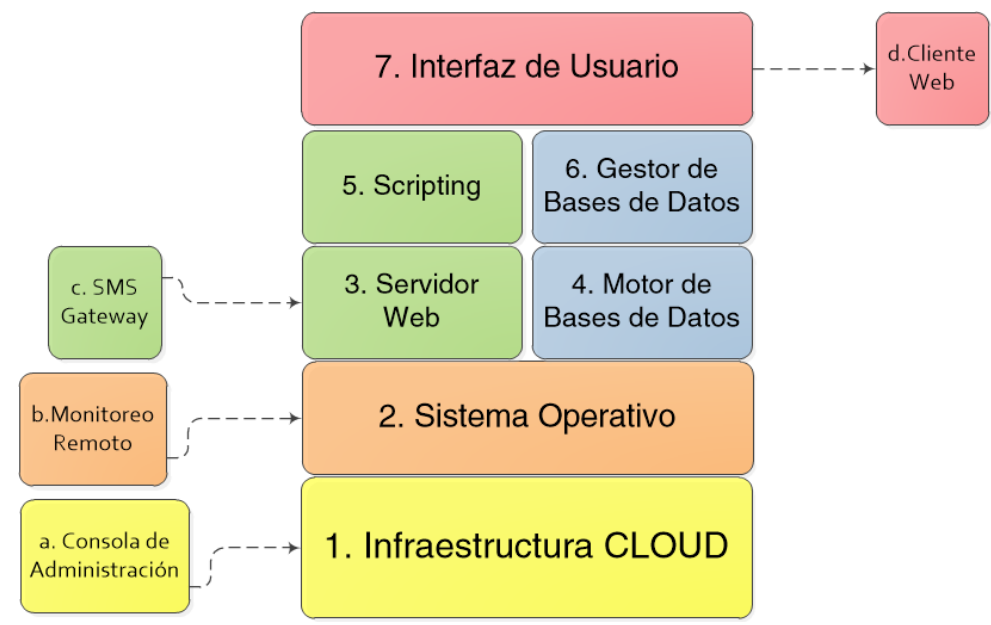

Figura 8. Infraestructura Lógica - Diseño de Software

Destaca en el planteamiento de la infraestructura la elección de un servidor basado en "Cloud Computing" o "Computación en la Nube", ya que si bien existen varias tecnologías de hardware disponibles, esta ha sido considerada como la más conveniente para el presente caso. Cloud Computing se impone a otras alternativas de alojamiento, como por ejemplo la utilización de un servidor dedicado; el cual evidentemente representaría un costo mucho mayor en la planificación del proyecto. Al contrario, la solución basada en la nube tiene como propósito principal reducir al mínimo los costos de implementación y mantenimiento, ya que su operación no requiere de instalaciones físicas, cableado, o suministro de energía eléctrica, además no es susceptible de obsolescencia como en el caso de un servidor dedicado. La computación en la nube también se impone a otra alternativa de alojamiento muy popular años atrás, el hosting convencional, gratuito o de pago, el cual permite montar recursos sobre un servidor previamente establecido, pero al tratarse de un medio compartido entre varios usuarios, tiene limitaciones de rendimiento, procesamiento y memoria, además de ser muy poco personalizable y escalable.

La plataforma de Cloud Computing seleccionada fue Amazon Web Services, AWS, que es el más grande comerciante de servicios de computación en la nube a nivel mundial, y cuya herramienta EC2, Elastic Computing Cloud, posibilita la implementación ágil de instancias o servidores virtuales dedicados, los cuales pueden ser arrancados remotamente en cuestión de minutos desde una consola de administración bastante intuitiva; EC2 también es compatible con la mayoría de sistemas operativos, y contrariamente a las dos alternativas anteriores, es totalmente escalable; en EC2 el usuario es quien decide aumentar o reducir recursos (servidores, memoria, núcleos de procesamiento, etc.), conforme vayan cambiando sus necesidades, además permite pagar solamente por la capacidad utilizada, reduciendo considerablemente los costos operacionales. Para la creación del presente proyecto se ha aprovechado la "capa gratuita de AWS", que faculta el uso de una instancia EC2 t2.micro sin costo por 12 meses.

Es importante destacar que contrariamente a la creencia popular, la computación en la nube es una tecnología bastante segura, para lo cual por supuesto se deben tomar las debidas 
previsiones, en nuestro caso particular todos los datos del servidor serán encriptados, y solo se podrá acceder a ellos si se posee una clave criptográfica de acceso, RSA (Rivest, Shamir, Adleman), la cual es proporcionada por EC2 al momento de crear una instancia por primera y única vez. Es tal el énfasis que hace Amazon Web Services en el aspecto seguridad, que sus servicios de Cloud Computing son utilizados por empresas de la envergadura de Netflix, Pfizer, Adobe, Siemens, Spotify, e instituciones como la NASA (Amazon, 2015).

Por otro lado, de la misma manera que en el diseño de hardware, con el objetivo de optimizar recursos, se tomó la decisión de utilizar software libre y gratuito a lo largo de todo el proceso de ejecución del proyecto. La Tabla 2 a continuación, muestra las herramientas que serán utilizadas para la implementación de cada elemento de la infraestructura lógica formulada anteriormente.

Tabla 2. Herramientas de Software Libre.

\begin{tabular}{|l|l|}
\hline Elemento de la Plataforma & Herramientas de Software \\
\hline Sistema Operativo del Servidor & Ubuntu Server \\
\hline Servidor Web & Apache Web Server \\
\hline Scripting & PHP \\
\hline Motor de Bases de Datos & MySQL Server \\
\hline Gestor de Bases de Datos & phpMyAdmin \\
\hline Interfaz Web & HTML5 + CSS3 \\
\hline Monitoreo Remoto & SSH Secure Shell \\
\hline SMS Gateway & Android App Based \\
\hline
\end{tabular}

Es evidente que la combinación de herramientas de software seleccionada no constituye la única alternativa en cuanto a las tecnologías disponibles para la creación de la plataforma, sin embargo cada una de ellas ha sido elegida por destacarse sobre otras soluciones, ya que han sido ampliamente probadas, documentadas, y están en continua evolución. A continuación se argumentan los pormenores de la elección de cada una de dichas herramientas.

Ubuntu Server. Se ha elegido el sistema operativo Ubuntu Server para alojar la plataforma por ser un sistema operativo confiable, seguro, robusto y de alto rendimiento. La edición Server de Ubuntu se gestiona totalmente desde un terminal, es decir, no dispone de una interfaz gráfica de usuario, o GUI (Graphic User Interface), lo que permite optimizar los recursos de hardware.

Apache Web Server. Se optó por Apache como servidor ya que a más de ser el líder indiscutible en el campo de las aplicaciones Web, se constituye como una solución completa, eficiente, segura, y personalizable, compatible con varios lenguajes de programación.

PHP. Los scripts encargados de automatizar las distintas funcionalidades de la plataforma serán implementados utilizando el lenguaje de programación orientado a la Web "PHP" (Hypertext Preprocessor), el cual se caracteriza por ser flexible, de alto rendimiento, optimizado para grandes demandas de tráfico; además, dado que PHP se ejecuta completamente en el lado del servidor, se garantiza que los usuarios no tengan acceso a información sensible contenida en los Scripts. 
MySQL Server. Se ha seleccionado MySQL, el gestor de bases de datos relacionales más utilizado alrededor del mundo, y uno de los de mejor rendimiento, dado que consume muy pocos recursos de hardware, reduciendo la carga de procesamiento en el servidor. MySQL cuenta además con uno de los motores de indexación (querys) más rápidos disponible en la actualidad.

phpMyAdmin. Si bien MySQL por si solo es una herramienta muy poderosa, la gestión de las bases de datos debe realizarse a través de su consola terminal, lo que es frecuentemente un procedimiento engorroso, se utilizará por lo tanto phpMyAdmin, una herramienta gráfica que simplifica la gestión de MySQL.

HTML5 + CSS3. La accesibilidad del usuario es uno de los aspectos más importantes en el diseño del proyecto, por lo tanto es imperativa la creación de una interfaz limpia, intuitiva, y eficiente, para lo cual se utilizará el lenguaje estándar de elaboración de páginas web HTML5 (HiperText Markup Language 5) junto con el lenguaje de estilos CSS3 (Cascading Style Sheets 3), los cuales se ejecutan de forma nativa en cualquier navegador actual, inclusive en dispositivos móviles.

SSH Secure Shell. Debido a que el servidor estará alojado en la nube y no se dispondrá de él físicamente, la única forma de gestionarlo y monitorearlo es a través de una conexión remota (encriptada) para lo cual se utilizará la herramienta SSH (Secure Shell) a través de un terminal.

SMS Gateway. Si bien existen equipos de hardware dedicados a cumplir esta función específica, son demasiado costosos, por lo que se ha decidido utilizar un SMS Gateway basado en software, específicamente la aplicación del mismo nombre creada para el sistema operativo móvil Android®, la cual es liviana, eficiente, segura, y tiene la capacidad de trabajar en tiempo real.

\subsection{Implementación}

A partir del diseño establecido para la plataforma se procederá a su implementación. El primer elemento que se pondrá en funcionamiento es el SMS Gateway, para lo cual se instaló la aplicación del mismo nombre en el dispositivo móvil encargado de recibir los pagos P2P, a continuación se realizaron las configuraciones de red del servidor remoto, es decir, se asignó la dirección IP, el puerto de conexión, y el Script PHP encargado de procesar la información.

El siguiente punto fue el establecimiento del servidor LAMP (Linux, Apache, MySQL, PHP) alojado en la nube, para lo cual se creó una cuenta de usuario en Amazon Web Services, y través de su consola de gestión se implementó una instancia EC2 tipo t2.micro que alojará el servidor LAMP. Una instancia t2.micro dispone de 1 unidad de procesamiento Intel Xeon® dedicada a servidores con 8 núcleos y turbo hasta $3.3 \mathrm{GHz}, 1$ GB de Memoria RAM, 1 conexión de red de 10 Gbps, y capacidad de carga variable por ráfagas optimizada para aplicaciones web (Amazon, 2015). Con la instancia EC2 puesta en funcionamiento, a través de una conexión SSH, se estableció un 
enlace remoto con el sistema operativo del servidor, desde cuyo terminal se realizó la instalación tanto de LAMP como del gestor gráfico phpMyAdmin, directamente desde los repositorios.

Luego de realizar la instalación y configuración de todos componentes de software, se procedió a la implementación de la base de datos MySQL utilizando la interfaz del gestor phpMyAdmin. Se creó cada tabla con sus respectivos campos, validando los tipos de datos, estableciendo las claves primarias, foráneas, e índices únicos, procurando siempre evitar la redundancia en su estructura lógica. El diagrama de la Figura 9 muestra la estructura de la base de datos:

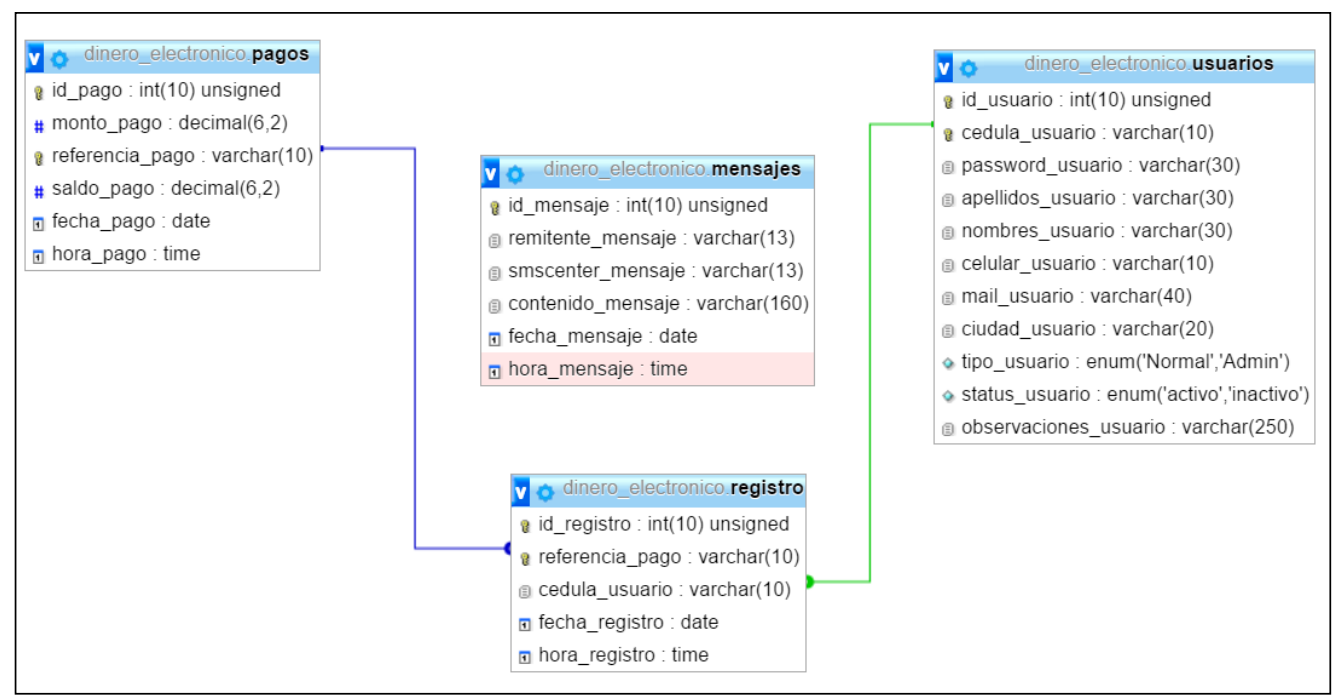

Figura 9. Estructura de la Base de Datos

Posteriormente se llevó a cabo la creación de los scripts PHP, encargados de ejecutar las distintas funcionalidades de la plataforma. Con el objetivo de simplificar la implementación del código, se diseñaron previamente diagramas de flujo que describen la lógica de funcionamiento de cada tarea, de esta forma se estableció con claridad el proceso de codificación posterior, así como las variables involucradas en el mismo. La Figura 10 muestra como ejemplo, el diagrama de flujo que describe la lógica de operación del Script PHP "registro de pagos".

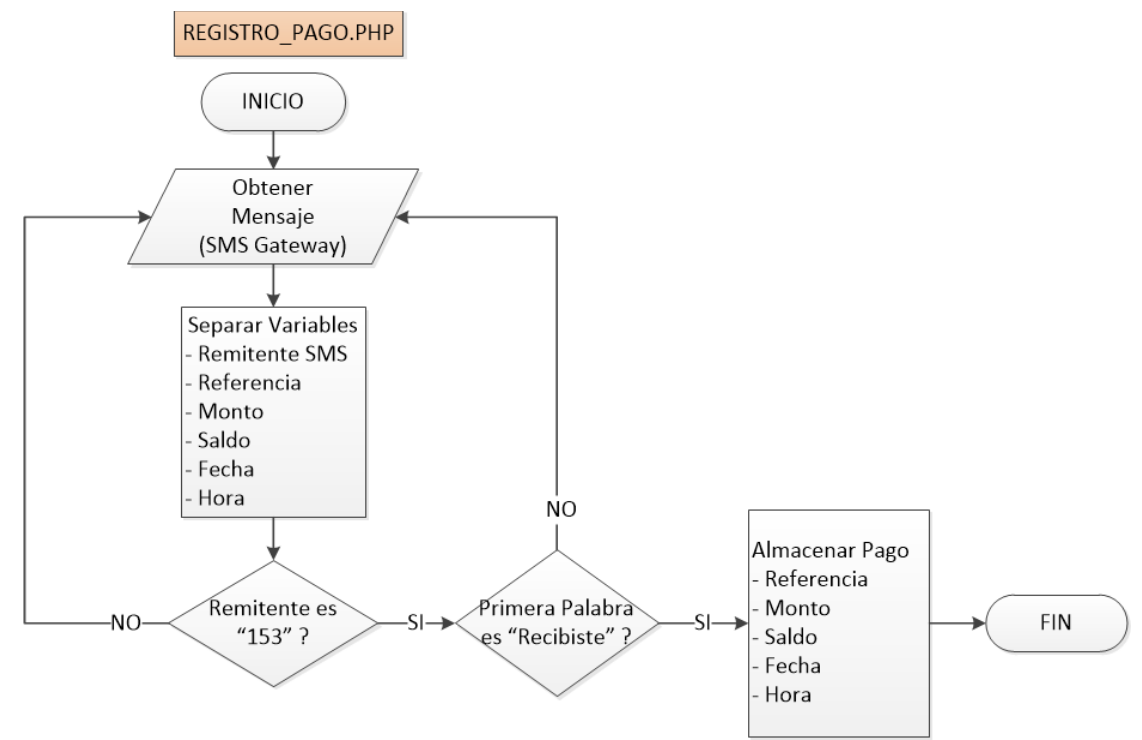

Figura 10. Diagrama de Flujo del Script "Registro de Pago". 
Como aspecto final se implementó la interfaz web de usuario, integrando todas las funcionalidades que componen la plataforma. El código de la interfaz fue escrito en HTML5 y CSS3 utilizando un editor de textos convencional. La plataforma creada fue nombrada "Denarium" y está conformada por las secciones ilustradas en la Figura 11 a continuación:

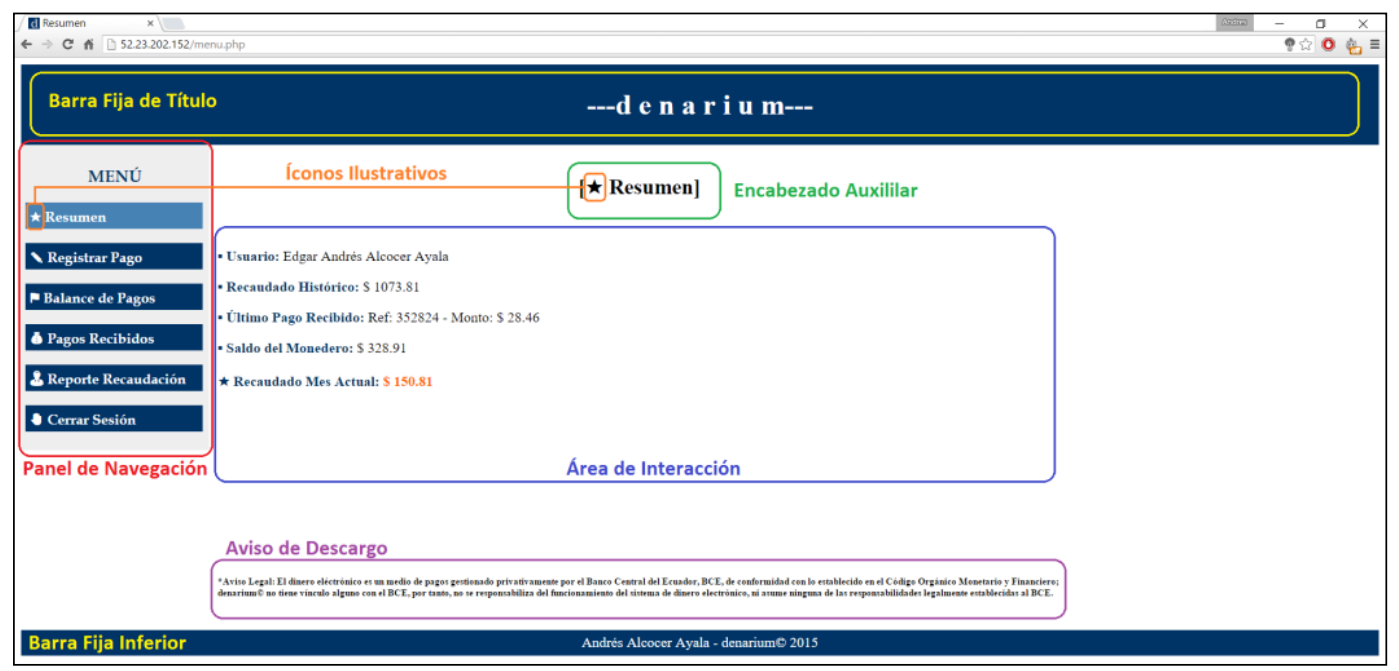

Figura 11. Interfaz de Usuario Plataforma "Denarium"

\section{Resultados}

Con el objetivo de evaluar el rendimiento de la plataforma se llevará a cabo su validación, que consiste en desarrollar y ejecutar pruebas específicas, las cuales se determinarán a partir de dos enfoques. En primer lugar se realizarán pruebas exclusivamente funcionales, dirigidas a analizar la experiencia del usuario final, sin tomar en cuenta la lógica interna o el desempeño individual de cada componente. El segundo enfoque comprende un análisis más relativo del rendimiento de la plataforma utilizando herramientas de gestión y monitoreo proporcionadas por AWS.

En la Figura 12 a continuación se muestra el resultado de ejecutar una prueba, la consulta de los pagos recibidos desde la interfaz de administrador; en esta se detallan los pagos recibidos con su correspondiente información. La Tabla 3 enumera el resto de funcionalidades validadas.

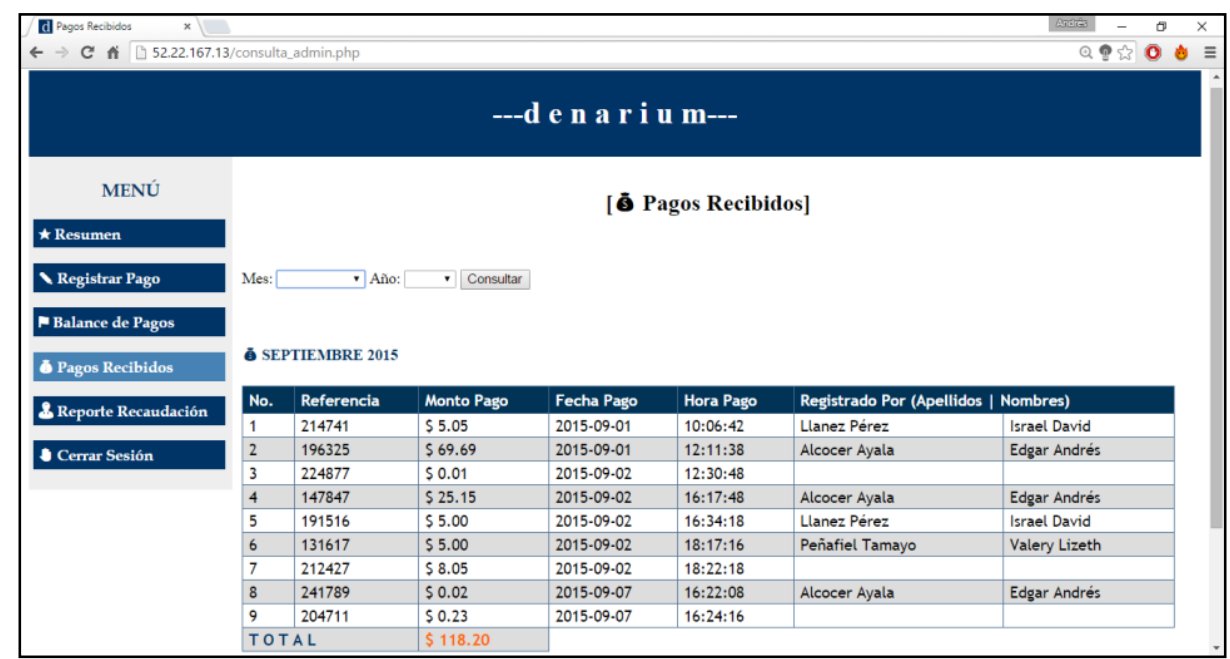

Figura 12. Pagos Recibidos - Interfaz Administrador. 
Tabla 3. Pruebas de Validación Funcional

\begin{tabular}{|c|c|c|}
\hline \# & FUNCIONALIDAD & RESULTADOS \\
\hline 1 & $\begin{array}{l}\text { Inicio de sesión (Usuario Normal y } \\
\text { Administrador) }\end{array}$ & $\begin{array}{l}\text { Si las credenciales de inicio de sesión son correctas, se accede a } \\
\text { la plataforma y se presenta el panel de navegación según el tipo de } \\
\text { usuario, caso contrario se muestra un mensaje de error. }\end{array}$ \\
\hline 2 & $\begin{array}{l}\text { Recibir un pago (Automático } \\
\text { Plataforma) }\end{array}$ & $\begin{array}{l}\text { Los pagos se registran inmediatamente en el sistema tras recibir el } \\
\text { SMS de confirmación de una transacción P2P. }\end{array}$ \\
\hline 3 & $\begin{array}{l}\text { Registrar un pago recibido a } \\
\text { nombre de un usuario. (Usuario } \\
\text { Normal y Administrador) }\end{array}$ & $\begin{array}{l}\text { Opción "Registrar Pago". Se solicita el ingreso del código de } \\
\text { referencia de la transacción, si este corresponde efectivamente a } \\
\text { un pago, se registra de forma automática a nombre del usuario, } \\
\text { caso contrario se muestra un mensaje de error. }\end{array}$ \\
\hline 4 & $\begin{array}{l}\text { Visualizar } \quad \text { balance } \\
\text { transacciones (Usuario Normal y } \\
\text { Administrador) }\end{array}$ & $\begin{array}{l}\text { Opción "Balance de Pagos". El usuario selecciona el mes y año } \\
\text { deseados, inmediatamente se despliega una tabla que contiene el } \\
\text { número de transacciones realizadas, los códigos de referencia } \\
\text { correspondientes, el valor de cada pago, la fecha y hora de } \\
\text { registro, así como el monto total de los valores registrados. }\end{array}$ \\
\hline 5 & $\begin{array}{l}\text { Visualizar un informe de los pagos } \\
\text { recibidos, y verificar a nombre de } \\
\text { quien están registrados. (Solo } \\
\text { Administrador) }\end{array}$ & $\begin{array}{l}\text { Opción "Pagos Recibidos". Luego de seleccionar el mes y año } \\
\text { deseados, se despliega una tabla con el detalle de TODOS los } \\
\text { pagos recibidos en la plataforma. De ser el caso se muestra } \\
\text { también el nombre del usuario que los registró. }\end{array}$ \\
\hline 6 & $\begin{array}{l}\text { Reporte de Recaudación (Solo } \\
\text { Administrador) }\end{array}$ & $\begin{array}{l}\text { Opción "Reporte de Recaudación". Tras seleccionar el mes y año } \\
\text { deseados, se despliega un resumen detallado de los montos } \\
\text { recaudados por cada usuario de la plataforma, así como el valor } \\
\text { total de los pagos registrados. }\end{array}$ \\
\hline 7 & $\begin{array}{l}\text { Resumen de } \text { Transacciones } \\
\text { (Usuario Normal y Administrador) }\end{array}$ & $\begin{array}{l}\text { Opción "Resumen". Permite visualizar un extracto abreviado de la } \\
\text { actividad de un usuario en la plataforma, este incluye su propio } \\
\text { nombre de usuario, el detalle del último pago registrado, el monto } \\
\text { recaudado del mes en curso, y el valor recaudado histórico. }\end{array}$ \\
\hline 8 & $\begin{array}{l}\text { Cerrar Sesión (Usuario Normal y } \\
\text { Administrador) }\end{array}$ & $\begin{array}{l}\text { La opción "Cerrar Sesión" bloquea el acceso a las funcionalidades } \\
\text { de la plataforma, y redirige el navegador a la página de inicio de } \\
\text { sesión. }\end{array}$ \\
\hline 9 & $\begin{array}{l}\text { Cierre Automático de Sesión } \\
\text { (Automático Plataforma) }\end{array}$ & $\begin{array}{l}\text { Si un usuario permanece } 10 \text { minutos seguidos en estado de } \\
\text { inactividad, la sesión de usuario es cerrada de manera automática, } \\
\text { con el objetivo de resguardar la seguridad de su información. }\end{array}$ \\
\hline
\end{tabular}

La siguiente prueba de validación consistió en monitorear el consumo de los recursos de procesamiento del servidor en un intervalo continúo de 6 horas, con una utilización del sistema de normal a moderada, realizada por un número de entre 1 y 23 usuarios ejecutando operaciones habituales. Como se observa en la Figura 13 la carga máxima alcanza el $0.25 \%$ de uso del procesador, y la carga mínima necesaria para que corra el sistema bordea el $0.04 \%$.

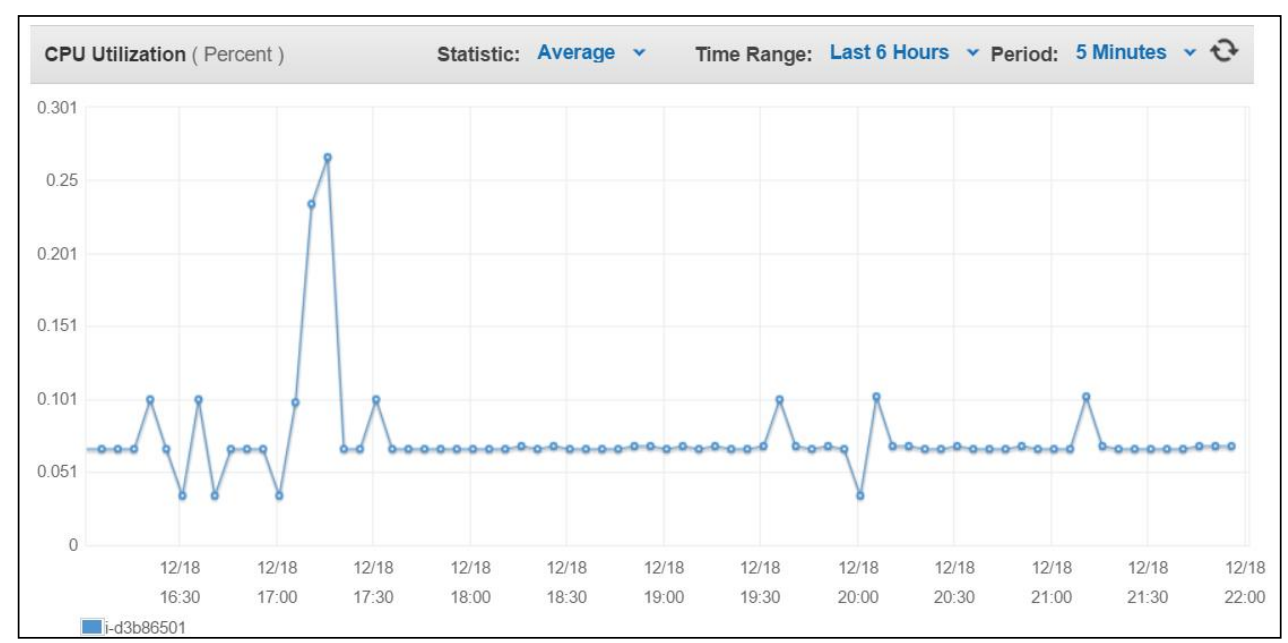

Figura 13. Carga de Procesamiento de la Plataforma vs Tiempo 


\section{Discusión}

Como se pudo observar la metodología prototyping permitió sistematizar el desarrollo de un prototipo funcional a partir de identificar una problemática existente, sin embargo, a pesar de ser deseable, por el momento no es posible comparar la plataforma Denarium con algún otro sistema, ya que la implementación del dinero electrónico en Ecuador es relativamente reciente y aún no se han desarrollado aplicaciones similares, o al menos estas no son de dominio público. No obstante, se espera que con el tiempo surjan otras innovaciones, que desde una perspectiva análoga permitan retroalimentar la idea inicial, y contribuir al desarrollo de útiles aplicaciones nuevas.

\section{Conclusiones y Recomendaciones}

Tras la ejecución de las pruebas de validación, se concluye que la plataforma cumple en la práctica con los requerimientos funcionales y de rendimiento, establecidos en el proceso de diseño, lo cual ratifica la elección de la metodología prototyping como una alternativa conveniente para el desarrollo del presente proyecto.

La utilización de la tecnología "Cloud Computing" como alternativa para la implementación del hardware, junto con el uso de herramientas de software libre, permitieron que la plataforma haya sido establecida en relativamente poco tiempo, y se constituya como una solución eficiente, segura, robusta, y de bajo costo.

La plataforma de gestión implementada proporciona una herramienta externa, que amplía las capacidades actuales del sistema de dinero electrónico del BCE, ya que permite a cualquier negocio o persona llevar un registro automático de sus transacciones $\mathrm{P} 2 \mathrm{P}$, así como generar balances de forma rápida y confiable.

A pesar de que según los datos obtenidos a partir de las pruebas de validación, el consumo de recursos de procesamiento de la plataforma, CPU, señala un valor demasiado bajo (entre $0.04 \%$ y $0.25 \%$ ), el sistema no fue sobredimensionado. Esto se explica sencillamente al esclarecer que las instancias EC2 de Amazon Web Services, están optimizadas para soportar un gran volumen de conexiones simultáneas, en nuestro caso específico la instancia básica t2.micro, soporta varios miles de ellas.

Se recomienda tomar el desarrollo del presente proyecto como fundamento para la creación de sistemas personalizados de gestión de pagos con dinero electrónico, por ejemplo para el diseño de una plataforma de venta de tickets de transporte o de cine.

Se recomienda no considerar la finalidad de este proyecto como la simple implementación de un sistema inalterable, sino como el preámbulo de la creación de un sinnúmero de nuevas herramientas y emprendimientos, que permitan simplificar la forma en que los ecuatorianos realizamos nuestras transacciones hoy en día. 


\section{Bibliografía}

Amazon. (2015). Amazon Web Services. Obtenido de Studie Cases: https://aws.amazon.com/es/solutions/case-studies/government-education/

ARCOTEL. (2015). Estadísticas: Líneas celulares activas en Ecuador. Obtenido de http://www.arcotel.gob.ec/estadisticas/

Asamblea Nacional del Ecuador. (2014). Código Orgánico Monetario y Financiero. Quito: Registro Oficial No.332.

Banco Central del Ecuador. (2015). Como funciona el dinero electrónico. Obtenido de http://www.dineroelectronico.ec/index.php/como-funciona/como-funciona-el-dineroelectronico

Banco Central del Ecuador. (2015). Como han evolucionado los medios de pago. Obtenido de http://www.dineroelectronico.ec/index.php/dineroelectronico/como-han-evolucionado-losmedios-de-pago

Beltrán, G. E. (2014). Dinero Electrónico como Herramienta de Inclusión Financiera en Ecuador. Quito: USFQ.

Chao, M. S., \& Ruiz, J. D. (2014). Análisis del sistema de dinero electrónico (SDE) implementado por el Banco Central del Ecuador y su posible impacto en la economía local. Guayaquil: Universidad Católica Santiago de Guayaquil.

Guadamuz, A. (2003). Electronic Money: A viable payment system? Edinburgh: University of Edinburgh.

Junta de Regulación Monetaria y Financiera. (2014). Normas para la gestión de Dinero Electrónico. Quito: Resolución No.005-2014-M.

M2M Money Payments Latam. (2015). Ecuador: Más de 45 mil cuentas activas de dinero electrónico. Obtenido de http://2015.mobilemoneylatam.com/

Ministerio Coordinador de Política Económica. (2013). Dinero electrónico un medio de pago sencillo, seguro y rápido. Ecuador Económico, 16-17. 